\title{
Comments on Randomly Sampled Non Local Means Image Filter
}

\author{
Alvaro Pardo \\ Department of Electrical Engineering, School of Engineering and Technologies, \\ Universidad Catolica del Uruguay \\ apardo@ucu.edu.uy
}

\begin{abstract}
In this work we comment the results presented in [1] regarding a random sampling approach of the Non Local Means (NLM) image denoising filter with respect to computational cost and denoising performance. We will show that although the approach is novel and mathematically revealing, the computation cost of the approach is higher, and the PSNR lower, compared to the classical version. Furthermore, we will present a probabilistic model to evaluate the performance of different versions of NLM and tune its parameters.
\end{abstract}

\section{Introduction}

The underlying idea of NLM is to filter the image using a weighted average of all pixels from it [2]. Given the pixel to be denoised, $i$, the weights $w_{i j}$ measure the similarity between patches centered at pixels $i$ and $j$ and the output of NLM filter is computed using equation (11). The trick is that corresponding patches are found all over the image imposing a non-local nature to the method. Similar ideas can be found in $3-5]$ and a review of several denoising strategies, including NLM, can be found in $[6]$ ]

One of the mayor drawbacks of NLM is its computational cost. If the image has $N$ pixels and the patches are of size $(2 K+1) \times(2 K+1)$ the computation cost of equation (11) has order $O\left(N^{2}(2 K+1)^{2}\right)$. The easiest way to reduce this cost is to use patches from a search region of size $(2 S+1) \times(2 S+1)$ around each pixel being processed. In this way, the cost is reduced to: $O\left(N(2 S+1)^{2}(2 K+1)^{2}\right)$. In this work we study the influence of the search region on the performance of NLM.

Recently, Chan, Zickler and Lu [1] introduced a novel interpretation of the NLM filter where the computation cost is reduced by randomly selecting a small set of patches to be used in (1). We will comment on the results presented in 1] and show that although the approach is indeed novel and mathematically revealing, the computation cost of the approach is higher than the classical version of NLM using a search window, and furthermore, the performance in terms of PSNR it is below the classical option. Therefore, we show that there are no clear benefits of the proposal with respect to computation cost or performance.

The same authors in 9] presented an extension of their work and introduce the idea of using an external database of patches, that is, not only patches from

E. Bayro-Corrochano and E. Hancock (Eds.): CIARP 2014, LNCS 8827, pp. 498 5052014.

(C) Springer International Publishing Switzerland 2014 
the image being processed. In this case, their proposal of random selection of candidate patches does present some novelty. In fact, the same idea is applied in recommender systems where random prototypes are used to find recommendations. In the area of recommender systems the distance between prototypes and the user for which recommendations are being computed it is known to play an important role on the results [10].

In this work we will borrow the idea of random selection of patches to study the performance of classical implementations of NLM and random NLM as presented in [1]. Furthermore, based on a probabilistic model we extract some results that can be used to guide the parameter selection for NLM.

\subsection{Non Local Means Image Filter}

NLM estimates the denoised value at pixel $i, \hat{I}_{i}$, using a weighted average of all pixels in the image [2]:

$$
\hat{I}_{i}=\frac{\sum_{j} w_{i j} I_{j}}{\sum_{j} w_{i j}}
$$

The weights $w_{i j}$ reflect the similarity between pixels $i$ and $j$ based on the distance between patches centered around them. Let $p_{i}$ and $p_{j}$ be the patches of size $(2 K+1) \times(2 K+1)$ centered at pixels $i$ and $j$ respectively. The restriction of the image $I$ to a patch $p_{i}$ will be denoted as $I\left(p_{i}\right)$, such that $I\left(p_{i}\right)=\left\{I_{k}, k \in p_{i}\right\}$. Using this vectors containing the image values the weights are computed as [2]:

$$
w_{i j}=\exp \left(-\left\|I\left(p_{i}\right)-I\left(p_{j}\right)\right\|_{2}^{2} / \sigma^{2}\right)
$$

Ideally, due to the non-local nature of the algorithm, similar patches are found across the whole image. This has two drawbacks. The first one, as we saw in the introduction, is the computational cost of searching similar patches across the whole image. The second one is related to the fact that taking weighted averages for all pixels in the image does not achieve the best PSNR (MSE). This issue was addressed in [6, 11] where the authors noted the problems with edge pixels. The problem is that in some cases the weights $w_{i j}$ are not able to discriminate between different patch classes. This is critical along edges since pixels along them have less corresponding patches in the image. Other authors that addressed the computational complexity of NLM encountered this trade off (for instance see [5]). Based on these considerations in [8] we conclude that a better solution is obtained via averaging only pixels within the same patch class. Therefore, the denoising performance depends also in a good patch classification. To conclude this section we point out that the performance of NLM depends also on the selection of the parameter $\sigma$.

\subsection{Random NLM}

The basic idea proposed by Chan et. al. [1] is to randomly select a subset of pixels to be used in equation (1). If $\left\{j_{1}, j_{2}, \ldots, j_{R}\right\}$ are $R$ indices randomly and 
uniformly selected from the whole set of indices $\{1, \ldots, N\}$ without replacement, the proposed variant for NLM is:

$$
\hat{I}_{i}=\frac{\sum_{t=1}^{R} w_{i j_{t}} I_{j_{t}}}{\sum_{t=1}^{R} w_{i j_{t}}} .
$$

In previous equation we dropped the pixel index since the same equation applies to all pixels.

The computational cost of this algorithm is $O\left(N(2 K+1)^{2} R\right)$. Clearly if $R>$ $(2 S+1)^{2}$ this cost will be higher than the one of using a search windows of size $(2 S+1) \times(2 S+1)$. The authors also presented experimental evidence, and a mathematical justification, showing that even for small values of $R$ the difference between the PSNR obtained with all pixels and the one obtained by their approach it is small. The mathematical model is indeed novel and opens new areas of research. For instance in [9] they extend their idea to image restoration using external databases.

However, as we mention before, the PSNR (MSE) obtained with (1) when using all patches in the images is below the maximum PSNR obtained for the classical NLM using a small search window. Secondly, in [1] the authors suggest that $R$ is set to $20 \%-30 \%$ of the total pixels which produces a computational cost that even for large search windows, large $S$, it is still well above the NLM that uses a search region. Hence, we are showing that there are no clear benefits on using this idea when dealing only with patches from the same image. When we use an external database of patches, as proposed in [9], random selection opens new opportunities. In fact in [9] methods for optimal selection of patches are discussed.

In next section we are going to present a model, inspired on the idea of random selection, which will enable us to compare the performance of the classical implementation of NLM (Classical NLM) that uses a search window around each pixel to be processed, a modified version that thresholds the weights to reduce the influence of dissimilar patches (Thresholded NLM) and the random selection version proposed by Chan et. al. in [1] (Random NLM).

\section{Performance Analysis}

Following the approach in [1] we are going to analyze the performance of the NLM filter assuming a random selection of the set of patches to be used.

We will consider that the set of randomly selected patches for pixel $i$ can be divided into two sets $A$ and $B$. $A$ contains all patches similar to patch centered at pixel $i, p_{i}$, and $B$ contains all selected patches not similar to $p_{i}$. We will assume that weights in set $A$ will be $w_{i} \approx 1$ while weights in $B$ will be small (small similarity). Therefore, our model is that $w_{i} \approx 1 \forall i \in A$ and $w_{i} \leq \epsilon \forall i \in B$, where $\epsilon$ is the parameter of the model which we will analyze later on. For each pixel $i$ restored intensity will be computed as:

$$
\hat{I}=\frac{\sum_{i \in A \cup B} w_{i} I_{i}}{\sum_{i \in A \cup B} w_{i}} .
$$


The best estimation is obtained when only similar patches from set $A$ are used for the NLM estimation. In this way we define the optimal estimation as:

$$
I_{A}=\frac{\sum_{i \in A} w_{i} I_{i}}{\sum_{i \in A w_{i}}}
$$

In order to estimate the errors that arise when both sets, $A$ and $B$, are used in the estimation we take the difference between (4) and (5):

$$
\hat{I}-I_{A}=I_{A}\left[\frac{\sum_{i \in B} w_{i}}{\sum_{i \in A} w_{i}+\sum_{i \in B} w_{i}}\right]+\frac{\sum_{i \in B} w_{i} I_{i}}{\sum_{i \in A} w_{i}+\sum_{i \in B} w_{i}} .
$$

Clearly the first term, that multiplies $I_{A}$, is the estimation error and the second term the bias. If the set $B$ is empty, or the weights in $B$ are close to zero, then both terms tend to zero. However, in most cases the set $B$ is not empty and the weights for patches in this set are not zero. That means that in equation (4) patches that are not good matches will influence the results deteriorating the estimation.

In what follows we describe the model for patches and weights in $A$ and $B$ and estimate the error and bias using monte carlo simulations. To simplify the model we will consider that $w_{i}=1$ for all patches in $A$. For patches in $B$ we model them as the results of a random sampling process. Weights in $B$ will be modeled as a random variable with an uniform distribution in $[0, \epsilon]\left(w_{i} \sim U_{[0, \epsilon]}\right)$ and the corresponding intensities for each patch are also modeled as uniform random variables in $[0,255]\left(I_{i} \sim U_{[0,255]}\right)$ independent of $w_{i}$.

Error simulation. Based on the previous model the error term, e, can be written as:

$$
e=\frac{\sum_{i \in B} w_{i}}{N_{A}+\sum_{i \in B} w_{i}}
$$

where $N_{A}$ is the number of patches in $A$. Dividing by numerator and denominator by $N_{A}$ we conclude that $e$ depends on the random variables $w_{i} / N_{A}$ (with uniform distribution in $\left.\left[0, \epsilon / N_{A}\right]\right)$ :

$$
e=\frac{\sum_{i \in B} w_{i} / N_{A}}{1+\sum_{i \in B} w_{i} / N_{A}}
$$

In Figure 1 we show the simulation results of $e\left(N_{B}\right)$ for different values of $\epsilon / N_{A}$. From this graph we can draw the following conclusions. First, even for small values of $\epsilon / N_{A}$, the error increases rapidly with $N_{B}$. This clearly shows that selecting a large number of patches is risky if the number of non-similar patches is not controlled. Secondly, smaller errors are achieved when $\epsilon$, the value that measures the similarity between non-corresponding patches, is small or when the number of similar patches, $N_{A}$, grows. Similarly, in Figure 1 we present the evolution of $e\left(N_{B}\right)$ when $N_{A}=100$. These results also show that even for small values of $\epsilon$ if $N_{B}$ is larger than $N_{A}$ then the error very easily goes above 0.1 . 
Now we are going to analyze the influence of $\epsilon$ in the error term. The sum of $N_{B}$ uniform random variables in $\left[0, \epsilon / N_{A}\right]$ has an Irwin-Hall distribution with mean $\frac{N_{B} \epsilon}{2 N_{A}}$. If the total of pixels in the search window is $N_{S}$ we can assume that a fraction of sampled patches are in set $A$ therefore $N_{A}=p N_{S}$ and $N_{B}=(1-p) N_{S}$ with $p \in[0,1]$. We can see that the mean of $w_{B}=\sum_{i \in B} w_{i}$ in equation (7) is independent of $N_{S}\left(\frac{N_{B} \epsilon}{2 N_{A}}=\frac{(1-p) \epsilon}{2 p}\right)$. In Figure 3 we show the results of simmulating the error term for diferrent values of $\epsilon$ and two values of $p$. When $N_{B}=0.5 N_{S}$ we are assuming that half of the points in the search window are not similar to the reference point. If we assume that for large search windows half of the pixels will be in $B$ we need to threshold the weights and consider only patches with similarities above $\epsilon=0.25$ to obtain an error below 0.1 .

The previous results, although they are based on a simple model, show that selecting a large number of patches without considering the distance to the reference patch will result in suboptimal performance of the NLM filter. If the weights do not correctly separate similar and non-similar patches, the results will be suboptimal too.

Bias simulation. The bias term $b$ can be expressed as:

$$
b=\frac{\sum_{i \in B} w_{i} / N_{A} I_{i}}{1+\sum_{i \in B} w_{i} / N_{A}} .
$$

Figure[2] shows the bias $b\left(N_{B}\right)$ varying $\epsilon / N_{A}$. Once again, even for small values of $\epsilon / N_{A}$ if $N_{B}$ increases so does the bias. In real applications it is difficult to reach a bias of 120 greylevels because usually we don't select $10^{6}$ patches. Nevertheless, these results show that selecting a large number of patches is dangerous if we cannot guarantee that selected ones are similar enough to the reference patch, $p_{i}$. In other words, expanding the search region or selecting a large number of patches without considering the similarity to the reference patch it is not optimal. Although expanding the search region might allow the inclusion of more candidates for set $A$, at the end of the day, if no control is applied during selection, the results tend to be suboptimal.

Figure 3 shows also the bias varying $\epsilon$ and confirms that to bound the bias a threshold on the weights is needed. We will come back to this point in next section.

\section{Experiments with Real Images}

In this section we compare Classical NLM, Thresholded NLM and Random NLM. In all cases the patches are of size $5 \times 5$ and the parameter $\sigma$ is set as suggested in [8]. The first experiment shows that the Random NLM performance is well below the one of Classical NLM. In Figure 4 we show the results for image House. Although as the number of selected candidates in Random NLM grows so does the performance, even when all the patches from the image are selected, 

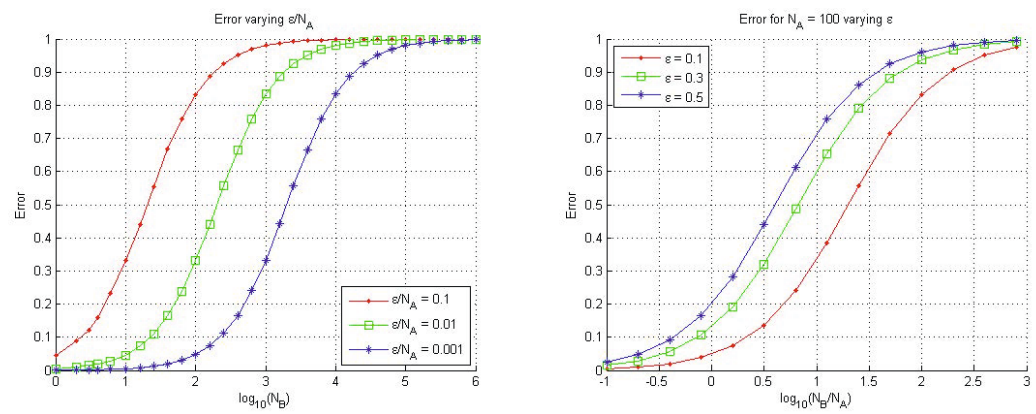

Fig. 1. Left: Installation of intrusive loops. Right: Computer Vision sensors.
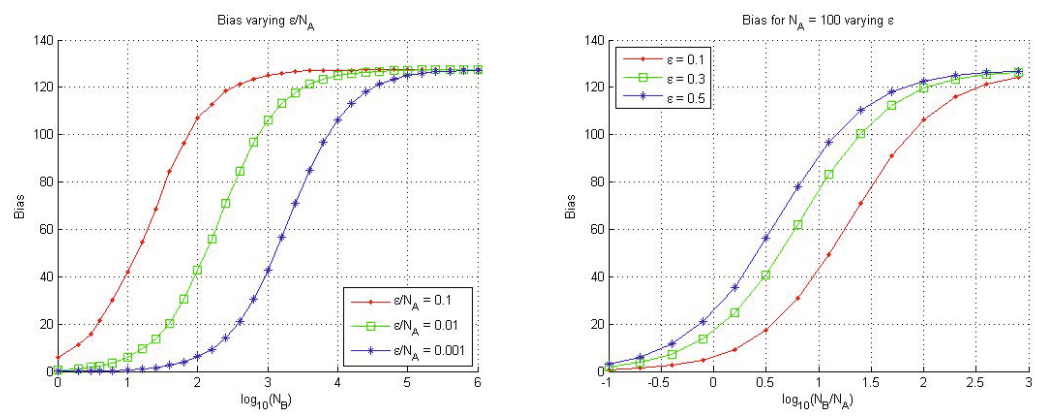

Fig. 2. Left: Installation of intrusive loops. Right: Computer Vision sensors.
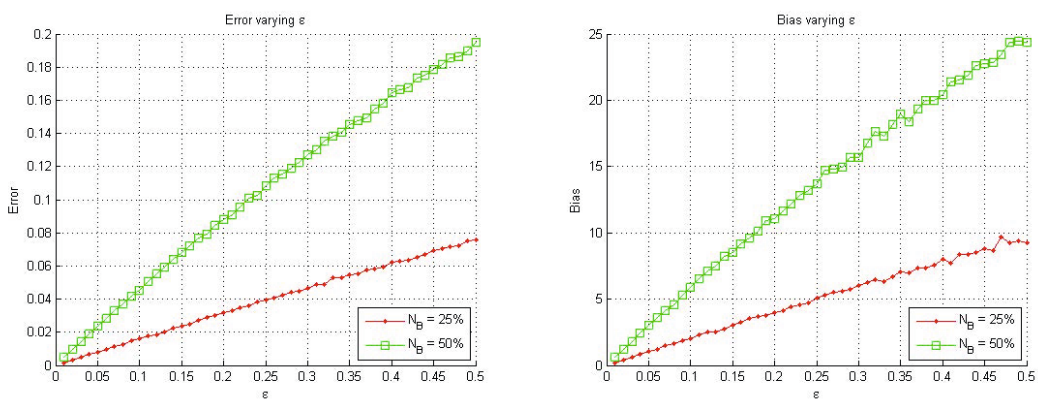

Fig. 3. Error (left) and bias (right) varying $\epsilon$ 

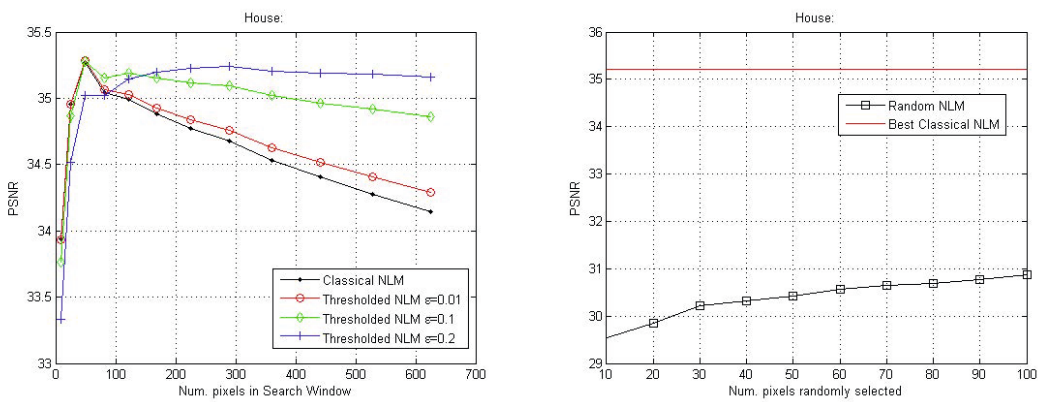

Fig. 4. Left: PSNR for image House using Classical NLM and Thresholded NLM. Right: PSNR for Random NLM compared to Classical NLM.
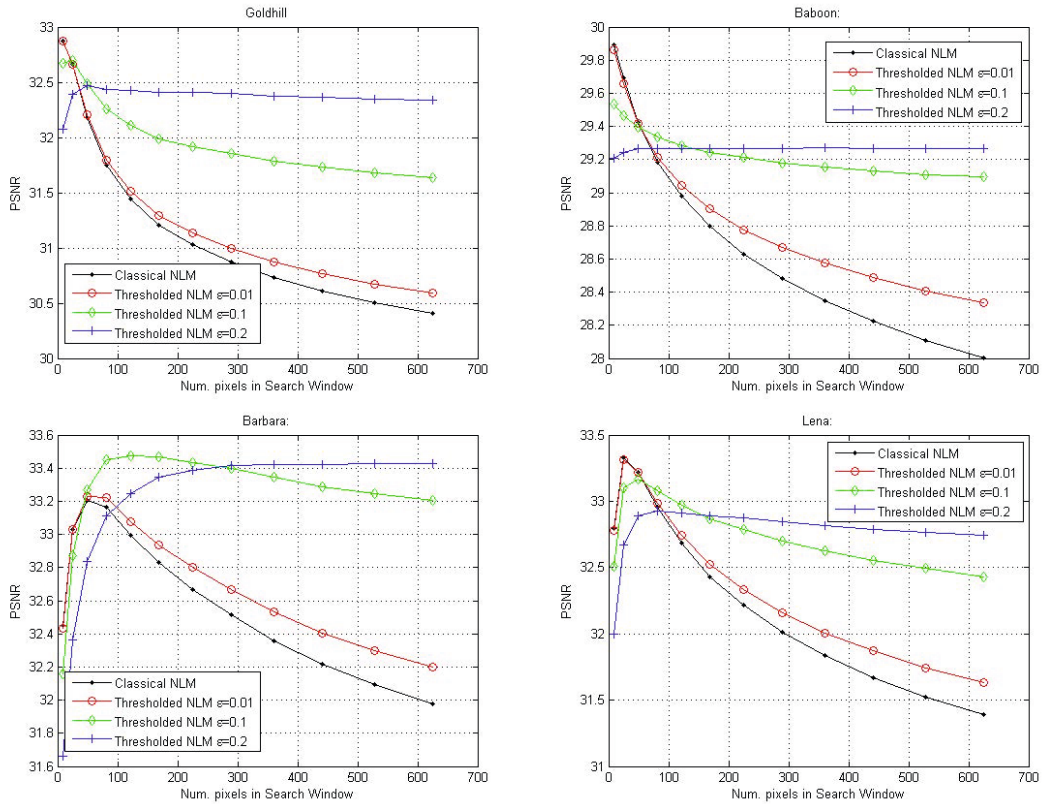

Fig. 5. PSNR for Classical NLM and Thresholded NLM for images Goldhill, Baboon, Barbara and Lenna

the performance is below the one obtained by Classical NLM. Furthermore, it can be seen that the performance of Classical NLM has a peak in performance for a search window of relatively small size. After the peak the performance drops very rapidly showing once again that selecting a large number of patches is suboptimal. Similar results are obtained in all the images tested and in Figure 5 we show the PNSR varying the search window size.

In Figure 5 we confirm that Classical NLM performances deteriorates when the number of pixels in the search window grows. On the other hand Threholded NLM is able to attain a stable PSNR while varying the size of the search window. As we can see when $\epsilon=0.2$ we obtain a very stable PSNR for all 
the tested images. This is in agreement with the conclusions obtained with our model in section 2. Therefore, we have an estimation of the threshold $\epsilon$ based on a probabilistic model. To best of our knowledge, this has never been addressed before.

\section{Conclusions}

In this work we showed that the method proposed in [1] does not provide improvements in terms of computation cost or PSNR with respect to Classical NLM. However, using the idea of random selection of patches, we proposed a model that allowed us to estimate the best values for the threshold in Thresholded NLM. The results of our model were confirmed in the experiments with real images.

\section{References}

1. Chan, S., Zickler, T., Lu, Y.: Fast non-local filtering by random sampling: it works, especially for large images. In: Proceedings of IEEE Conference on Acoustics, Speech and Signal Processing (ICASSP 2013), Vancouver, Canada, pp. 1603-1607 (2013)

2. Buades, A., Coll, B., Morel, J.: Denoising image sequences does not require motion estimation. In: Proc. IEEE Conf. on Advanced Video and Signal Based Surveillance, pp. 70-74 (2005)

3. Awate, S.P., Whitaker, R.T.: Unsupervised, information-theoretic, adaptive image filtering for image restoration. IEEE Trans. Pattern Anal. Mach. Intell. 28(3), 364$376(2006)$

4. Boulanger, J., Kervrann, C., Bouthemy, P.: Adaptive space-time patch-based method for image sequence restoration. In: Workshop on Statistical Methods in Multi-Image and Video Processing, SMVP 2006 (May 2006)

5. Mahmoudi, M., Sapiro, G.: Fast image and video denoising via nonlocal means of similar neighborhodds. IEEE Signal Processing Letters 12(12), 839-842 (2005)

6. Buades, A., Coll, B., Morel, J.: A review of image denoising algorithms, with a new one. SIAM Multiscale Modeling and Simulation 4(2), 490-530 (2005)

7. Buades, A., Coll, B., Morel, J.M.: The staircasing effect in neighborhood filters and its solution. IEEE Transactions on Image Processing 15(6), 1499-1505 (2006)

8. Pardo, A.: Analysis of non-local image denoising methods. Pattern Recognition Letters 32(16), 2145-2149 (2011)

9. Chan, S., Zickler, T., Lu, Y.: Monte carlo non local means: Random sampling for large-scale image filtering. Sunmitted to IEEE Trans. Image Processing (2014)

10. Zeng, C., Xing, C.X., Zhou, L.Z.: Similarity measure and instance selection for collaborative filtering. In: Proceedings of the 12th International Conference on World Wide Web, pp. 652-658. ACM (2003)

11. Bertalmio, M., Caselles, V., Pardo, A.: Movie denoising by average of warped lines. IEEE Transactions on Image Processing 16(9), 2333-2347 (2007) 